\title{
Spatial variation of the cooling lines in the Orion Bar from Herschel/PACS
}

\author{
J. Bernard-Salas ${ }^{1}$, E. Habart ${ }^{1}$, H. Arab ${ }^{1}$, A. Abergel ${ }^{1}$, E. Dartois ${ }^{1}$, P. Martin ${ }^{2}$, S. Bontemps ${ }^{3}$, C. Joblin ${ }^{4,5}$, \\ G. J. White ${ }^{6,7}$, J.-P. Bernard ${ }^{4,5}$, and D. Naylor ${ }^{8}$
}

\author{
1 Institut d'Astrophysique Spatiale, Paris-Sud 11, 91405 Orsay, France \\ e-mail: jbernard@ias.u-psud.fr \\ 2 Canadian Institute for Theoretical Astrophysics, Toronto, Ontario, M5S 3H8, Canada \\ 3 CNRS/INSU, Laboratoire d'Astrophysique de Bordeaux, UMR 5804, BP 89, 33271 Floirac Cedex, France \\ ${ }^{4}$ Université de Toulouse, UPS-OMP, IRAP, Toulouse, France \\ 5 CNRS, IRAP, 9 Av. colonel Roche, BP 44346, 31028 Toulouse Cedex 4, France \\ 6 Dept. of Physicics \& Astronomy, The Open University, Milton Keynes MK7 6AA, UK \\ 7 Space Science \& Technology Division, The Rutherford Appleton Laboratory, Chilton, Didcot OX11 0NL, UK \\ 8 Institute for Space Imaging Science, University of Lethbridge, Lethbridge, Canada
}

Received 13 September 2011 / Accepted 11 November 2011

\section{ABSTRACT}

\begin{abstract}
Context. The energetics in photo-dissociation regions (PDRs) are mainly regulated by the balance between the heating from the photo-electric effect acting on dust grains, and the cooling via the copious emission of photons in far-infrared lines. The Orion Bar is a luminous and nearby PDR, which presents to the observer an ideal edge-on orientation in which to study this energy balance. Spatially resolved studies of such a nearby system are essential as they enable us to characterise the physical processes that control the energetics of the regions and can serve as templates for distant systems where these processes cannot be disentangled.

Aims. We characterise the emission of the far-infrared fine-structure lines of [ $\left.\mathrm{C}_{\mathrm{II}}\right](158 \mu \mathrm{m}),\left[\mathrm{O}_{\mathrm{I}}\right](63$ and $145 \mu \mathrm{m})$, and [N $\left.\mathrm{II}\right](122 \mu \mathrm{m})$ that trace the gas local conditions, via spatially resolved observations of the Orion Bar. The observed distribution and variation of the lines are discussed in relation to the underlying geometry and linked to the energetics associated with the Trapezium stars.

Methods. Herschel/PACS observations are used to map the spatial distribution of these fine-structure lines across the Bar, with a spatial resolution between $4^{\prime \prime}$ and $11^{\prime \prime}$ and covering a total square area of about $120^{\prime \prime} \times 105^{\prime \prime}$. The spatial profile of the emission lines are modelled using the radiative transfer code Cloudy.

Results. The Herschel observations reveal in unprecedented detail the morphology of the Bar. The spatial distribution of the [C II] line coincides with that of the $\left[\mathrm{O}_{\mathrm{I}}\right]$ lines. The $\left[\mathrm{N}_{\text {II }}\right.$ ] line peaks closer to the ionising star than the other three lines, but with a small region of overlap. We can distinguish several knots of enhanced emission within the Bar indicating the presence of an inhomogenous and structured medium. The emission profiles cannot be reproduced by a single PDR, clearly indicating that, besides the Bar, there is a significant contribution from additional PDR(s) over the area studied. The combination of both the [ $\left.\mathrm{N}_{\mathrm{II}}\right]$ and [O I $145 \mu \mathrm{m}$ lines can be used to estimate the $\left[\mathrm{C}_{\mathrm{II}}\right]$ emission and distinguish between its ionised or neutral origin. We have calculated how much [C $\left.\mathrm{II}\right]$ emission comes from the neutral and ionised region, and find that at least $\sim 82 \%$ originates from the photo-dissocciation region. Together, the [C II] $158 \mu \mathrm{m}$ and [O I] 63 and $145 \mu \mathrm{m}$ lines account for $\sim 90 \%$ of the power emitted by the main cooling lines in the Bar (including $\mathrm{CO}, \mathrm{H}_{2}$, etc.), with [O I] $63 \mu \mathrm{m}$ alone accounting for $72 \%$ of the total.
\end{abstract}

Key words. ISM: lines and bands - H II regions - infrared: general - ISM: individual objects: Orion Bar

\section{Introduction}

Most of the mass in the interstellar medium (ISM) is in neutral form. Photo-dissociation regions (PDRs) are neutral regions of the ISM at the interface between the stars and opaque cores of molecular clouds, where the heating and chemistry are regulated by the penetrating far ultraviolet (FUV) photons of the ionising source(s) (e.g. Hollenbach \& Tielens 1999; Bernard-Salas \& Tielens 2005). The gas in the PDRs cools mainly via the emission of far-infrared (FIR) fine structure lines such as [C II], [O I], and molecules (e.g. $\mathrm{CO}, \mathrm{H}_{2}$ ). The intensity of these lines depends on local conditions in the gas, and when compared to PDR models can be used to trace the spatial evolution of the physical conditions of the gas across the PDRs.

Due to its proximity (415 pc, Menten et al. 2007) and edge-on orientation, the Orion Bar has been subject to extensive studies in the literature, where it is usually adopted as the prototypical PDR template in the study of high mass star forming regions, and is often used to test PDR models (e.g., Pellegrini et al. 2009). It is one of the brightest PDRs with an FUV radiation field at the ionisation front of $G=1-4 \times$ $10^{4} G_{0}$ (Tielens \& Hollenbach 1985; Marconi et al. 1998), where $G_{0}$ is the mean interstellar radiation field $\left(1.6 \times 10^{-6} \mathrm{~W} \mathrm{~m}^{-2}\right.$, Habing 1968). Tielens et al. (1993) showed how the Bar presented a layered distribution of polycyclic aromatic hydrocarbons (PAHs), $\mathrm{H}_{2}$, and $\mathrm{CO}$. This distribution is thought to be the result of an extended gas component seen edge-on, of average gas density between $10^{4}-10^{5} \mathrm{~cm}^{-3}$. Evidence of clumps have also been suggested to explain high density and temperature tracers (e.g., Burton et al. 1990; van der Werf 1996). Recently, Rubin et al. (2011) presented mid-IR Spitzer observations that extend beyond the Bar, from 2'1 to up to 12 '. 1 from the ionising $\operatorname{star} \theta^{1}$ Ori C. They detect ionised material $\left(\mathrm{Ne}^{+2}\right.$, IP of $\left.41 \mathrm{eV}\right)$ all the way up to the boundary of their observations. They also 
find evidence for a decrease in electron density and an increase of the PDR tracers ([Si II] $34.8 \mu \mathrm{m}$, [Fe II] $26.0 \mu \mathrm{m}$, and molecular hydrogen) as the distance from $\theta^{1}$ Ori $\mathrm{C}$ increases. In the FIR, Herrmann et al. (1997) mapped the Orion region with a 22" to $55^{\prime \prime}$ beam using the Kuiper Airborne Observatory (KAO). Their derived column densities in the $\mathrm{Bar}$ for $\mathrm{O}^{0}$ and $\mathrm{C}^{+}$were in agreement with an edge-on geometry.

In this paper, we present the first maps of the [C $\mathrm{II}](158 \mu \mathrm{m})$, [O I] (63 and $145 \mu \mathrm{m})$, and [N II] $(122 \mu \mathrm{m})$ lines of the Orion Bar region from the recently launched Herschel Space Observatory (HSO, Pilbratt et al. 2010). With its access to FIR and superb spatial resolution at these wavelengths, the HSO allows us to study the spatial distribution of these lines in unprecedented detail and so improve our understanding of the ionised and neutral interface. These observations enable us to trace the spatial evolution and excitation conditions of the gas across the illuminated interface of the Bar. This paper is part of a Herschel study of the Orion Bar by our group and is complemented by Herschel/SPIRE studies of the $\mathrm{CO}$ and $\mathrm{CH}^{+}$line emission (Habart et al. 2010; Naylor et al. 2010), and dust emission (Arab et al., in prep.).

The paper is organized as follows. The observations, data reduction and line measurements are given in Sect. 2. In the following section (Sect. 3) the spatial morphology of the lines is discussed. In Sect. 4 the distribution and correlation of the lines are analysed. Section 5 presents a model tailored to reproduce the distribution of the lines. Discussions on the origin of the [C II] emission and the cooling are presented in Sects. 6 and 7. Finally, the conclusions are summarized in the last section.

\section{Observations and data reduction}

The observations were taken using the PACS instrument (Poglitsch et al. 2010) on board the HSO on 24 February 2010. These observations are part of the Evolution of the Interstellar Medium guaranteed time key project (observation ID = 1342 191 152) (Abergel et al. 2010). Four fine-structure lines were targeted: [C II] at $158 \mu \mathrm{m}$, [O I] at 63 and $145 \mu \mathrm{m}$, and $\left[\mathrm{N}_{\mathrm{II}}\right]$ at $122 \mu \mathrm{m}$. At these wavelengths, and in increasing order, PACS provides a spectral resolution of $3420,1050,1170$, and 1270 respectively $\left(v \sim 90-285 \mathrm{~km} \mathrm{~s}^{-1}\right)$. The observations were taken during science demonstration phase (SDP) in the - now decommissioned - wavelength switching mode.

To trace the PDR and $\mathrm{H}_{\text {II }}$ region interface a $4 \times 4$ raster map was performed. Each raster position (or footprint) is composed by $5 \times 5$ spatial pixels referred to as spaxels. For each spaxel the line is observed in 16 different spectral scans, each with an up and down scan. The configuration at the time of the observation is shown in Fig. 1, where the raster map at the epoch of observation is overlaid on top of an $8 \mu \mathrm{m}$ IRAC image of the Orion Bar. We note that an additional map in the direction of the Trapezium stars and overlapping the current observation is currently scheduled for observation and will be presented in a future paper. Given the brightness of the lines, the minimum exposure configuration of one cycle and repetition per line was performed. For these maps the best sampling is achieved using Nyquist sampling, which we adopted for the lines measured in the red channel ([C II] at $158 \mu \mathrm{m}$, [O I] $145 \mu \mathrm{m}$, and [N II] at $122 \mu \mathrm{m})$. This consists of a raster point and raster line step of $24{ }^{\prime \prime} 0$ and $222^{\prime \prime} 0$ respectively. This results approximately in steps of $2 / 3$ and $1 / 2$ of the slit size along both directions. For the [O I] $63 \mu \mathrm{m}$ line located in the red channel, Nyquist sampling is achieved with $16{ }^{\prime \prime} 0$ and $144^{\prime \prime} 5$ raster point and line steps. However, to minimise exposure time, and given the strength of the line, the same step

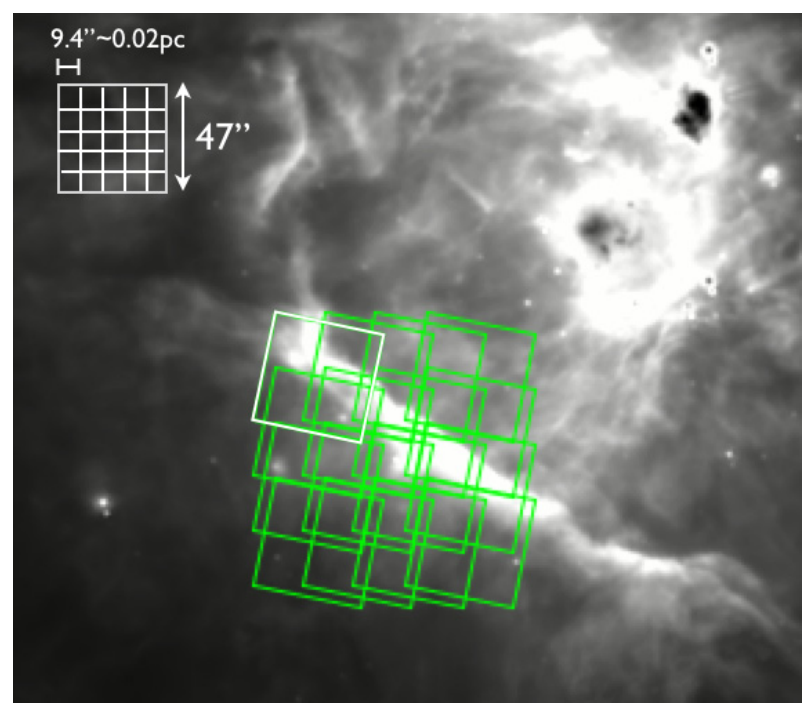

Fig. 1. Overlay of the [O I] $63 \mu \mathrm{m}$ PACS observation on a Spitzer/IRAC $8 \mu \mathrm{m}$ image of the Orion Bar. A $4 \times 4$ raster map -16 overlapping footprints - was performed (see Sect. 2). An example of a footprint with its $5 \times 5$ spaxels is illustrated on the top left of the figure together with their dimensions. The map covers the region before and behind the Bar (toward $\theta^{1}$ Ori C).

size as the lines for the red channel was adopted. An off position, about $40^{\prime}$ from the center of the map, was taken at the beginning and the end of the observation (not shown in the figure). This observation is not subtracted from the data as the purpose of the wavelength switching mode is to cancel out the background by determining a differential line profile. The off observation, however, confirmed that there was no background contamination to any of the lines studied.

The data were processed using version 6.1 .0 of the reduction and analysis package HIPE (Ott 2010). From level 0.5 to level 1 the standard procedures were followed. From this level on the cubes were further processed, using proprietary tools, to correct for drifting and for flux misalignments between scans. Drifting is caused by temperature deviations in the telescope which can cause the signal to be modified over time. They can be easily identified by comparing the up and down scans. To correct for this effect we first passed a median filter in the time domain to avoid glitches. The continuum was then fitted using a linear fit ${ }^{1}$ to characterise and remove the drift. The flux misalignment between the spectral scans can be due to improper dark subtraction or flatfield correction. The former will have an additive effect, and the latter a multiplicative one. In our case, the scans could be well aligned using an additive value which indicates that in our observations the cause of the flux misalignments is the dark subtraction. We note that the lines are very strong and these corrections were minor.

At this point the cubes are exported and we used the IDL-based software PACSman (Lebouteiller et al., in prep.) to measure the line fluxes (by fitting a Gaussian) and create the final map. In this code, the line fluxes are measured for all the spaxels independently. The lines are not spectrally resolved. To create the final map, PACSman recreates an oversampled pixelated grid of the observations (with $3^{\prime \prime}$ pixel resolution) and calculates the average fractional contribution of the given spaxels to the relevant position. The code calculates the statistical uncertainties

\footnotetext{
${ }^{1}$ In our data the signal was either linear or slightly curved. A polynomial fit of second degree produced very similar results.
} 


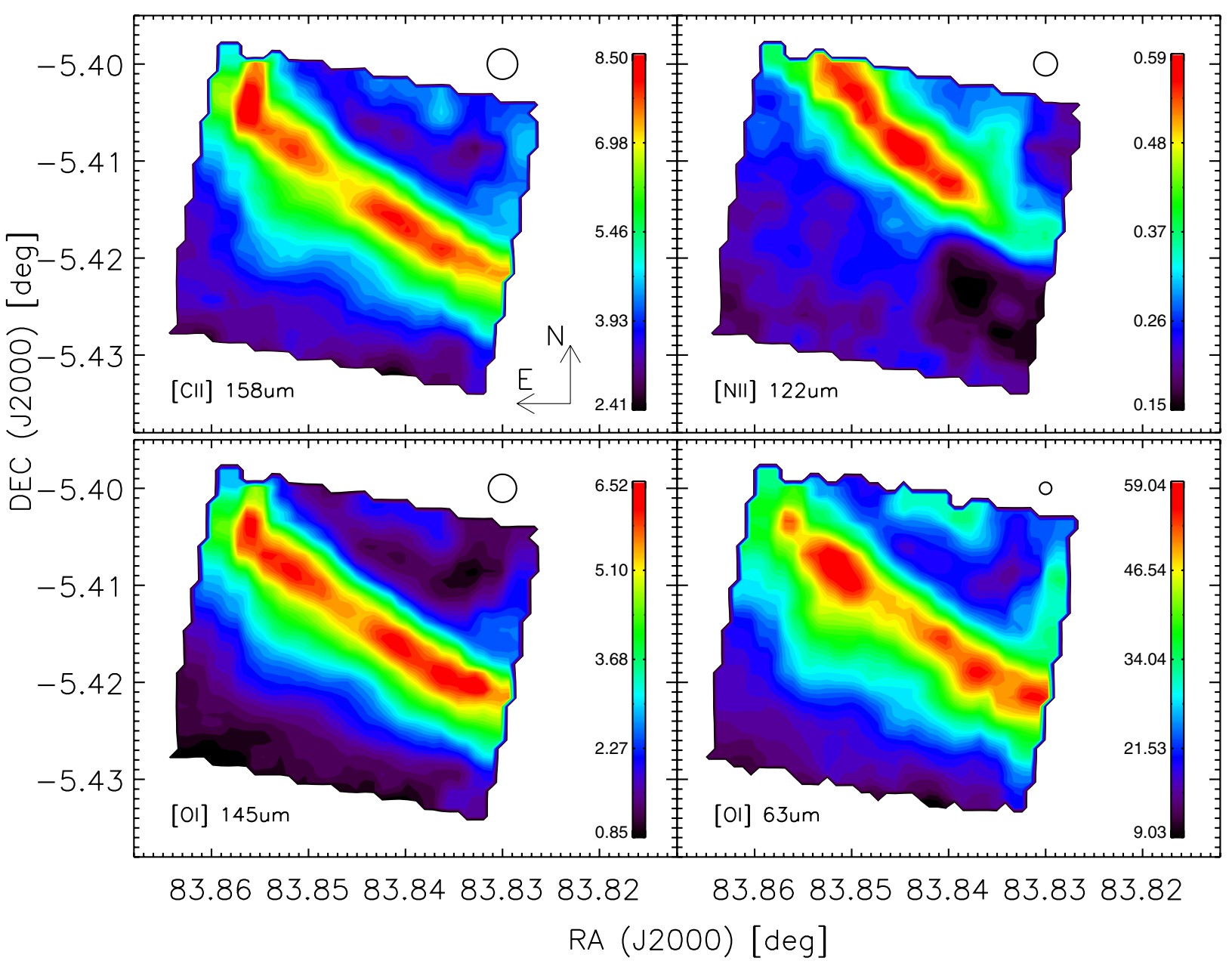

Fig. 2. PACS contour maps of the [C $\mathrm{II}](158 \mu \mathrm{m})$, [O I] $(63$ and $145 \mu \mathrm{m})$, and [ $\mathrm{N}_{\mathrm{II}}$ ] $(122 \mu \mathrm{m})$ lines in units of $10^{-6} \mathrm{~W} \mathrm{~m}^{-2} \mathrm{sr}^{-1}$. In increasing order of wavelength the beams sizes are $4 .{ }^{\prime \prime}, 8,8^{\prime \prime} 8,10^{\prime \prime}$, and $11^{\prime \prime}$. Note the offset from zero in the intensity scale to emphasize the detail.

on the fly, which include the dispersion in the reduction process and the rms of the fit. These uncertainties are small and usually amount to less than $4 \%, 1 \%, 3 \%$ for the $\left[\mathrm{C}_{\mathrm{II}}\right.$ ] and [O I lines. For the weaker [N II] $122 \mu \mathrm{m}$ line, these are higher and oscillate between $5-13 \%$. The relative accuracy between spaxels given in the manual is $10 \%^{2}$, and for the remaining of the paper we have adopted the higher of this value or the uncertainty given by PACSman. We note that absolute flux calibration is quoted to be $30 \%$ of the peak-to-peak accuracy (scatter around the expected flux densities). The final maps for each of the four lines are shown in Fig. 2. We have compared our [ $\left.\mathrm{C}_{\mathrm{II}}\right]$ and $\left[\mathrm{O}_{\mathrm{I}}\right]$ line fluxes with those measured by Herrmann et al. (1997) with the Fabry-Pérot interferometer FIFI and integrated over a 55" beam. Their measurements are in good agreement (within $~ 30 \%$ ) with those reported in this paper when convolved to their larger beam size.

\section{Spatial distribution}

The observed spatial distribution of the lines is shown in Fig. 2, where the ionising star $\theta^{1}$ Ori $\mathrm{C}$ is illuminating the region from the top right, outside the maps (see also Fig. 4a for a sketch of the different regions). The beams sizes are represented in the top

\footnotetext{
${ }^{2}$ From the PACS spectroscopy performance and calibration manual. This can be found at http://herschel.esac.esa.int/twiki/ bin/view/Public/PacsCalibrationWeb
}

right of each map and correspond, in increasing order of wavelength, to $4{ }^{\prime \prime} 5,8^{\prime \prime} 8,10^{\prime \prime}$, and $11^{\prime \prime}$. At the distance of the Bar $(415 \mathrm{pc}), 10^{\prime \prime}$ corresponds to a physical scale of $0.02 \mathrm{pc}$. The $\mathrm{Bar}^{3}$ is resolved in all four lines and we detect emission all over the region probed, including detection of [N $\mathrm{NI}$ (ionised gas) behind the Bar. The range of intensities is not large, with variations by factors 4 ([C II $]$ and $[\mathrm{N} \mathrm{II}]$ ) to 8 ([O I $]$ ) from the peak (Bar) to the fainter (outer) regions.

The overall morphology of the region can be best discussed from the [O I] $63 \mu \mathrm{m}$ map because its smaller point spread function (PSF) provides the highest detail (Fig. 2). Even if this line is more affected by self-absorption, the gain in resolution bests this caveat. The emission peaks at about $123^{\prime \prime}$ SE of $\theta^{1}$ Ori C. The most striking feature is the presence of several knots of enhanced emission resulting in small scale structures, which suggests a clumpy distribution within the Bar. These knots, three in the south-west and the two in the north-east, are bridged by weaker emission, $\sim 16 \%$ lower in flux than the knots. In the [OI] $63 \mu \mathrm{m}$ image, the smaller knots have diameters between $\sim 6^{\prime \prime}$ and $\sim 10^{\prime \prime}$ which, at the distance of the Orion nebula, correspond to $0.01-0.02 \mathrm{pc}$. The smaller ones $\left(6^{\prime \prime}\right)$ are marginally

${ }^{3}$ For the remainder of the paper, the Bar is defined as the region where the emission is higher than $75 \%$ of the peak emission for carbon. This threshold falls between the third and fourth highest contours in Fig. 3 and is represented by the solid line in Fig. 4a. Similarly, we use the same definition for the discussion in Sect. 6 about the [N II] peak. 


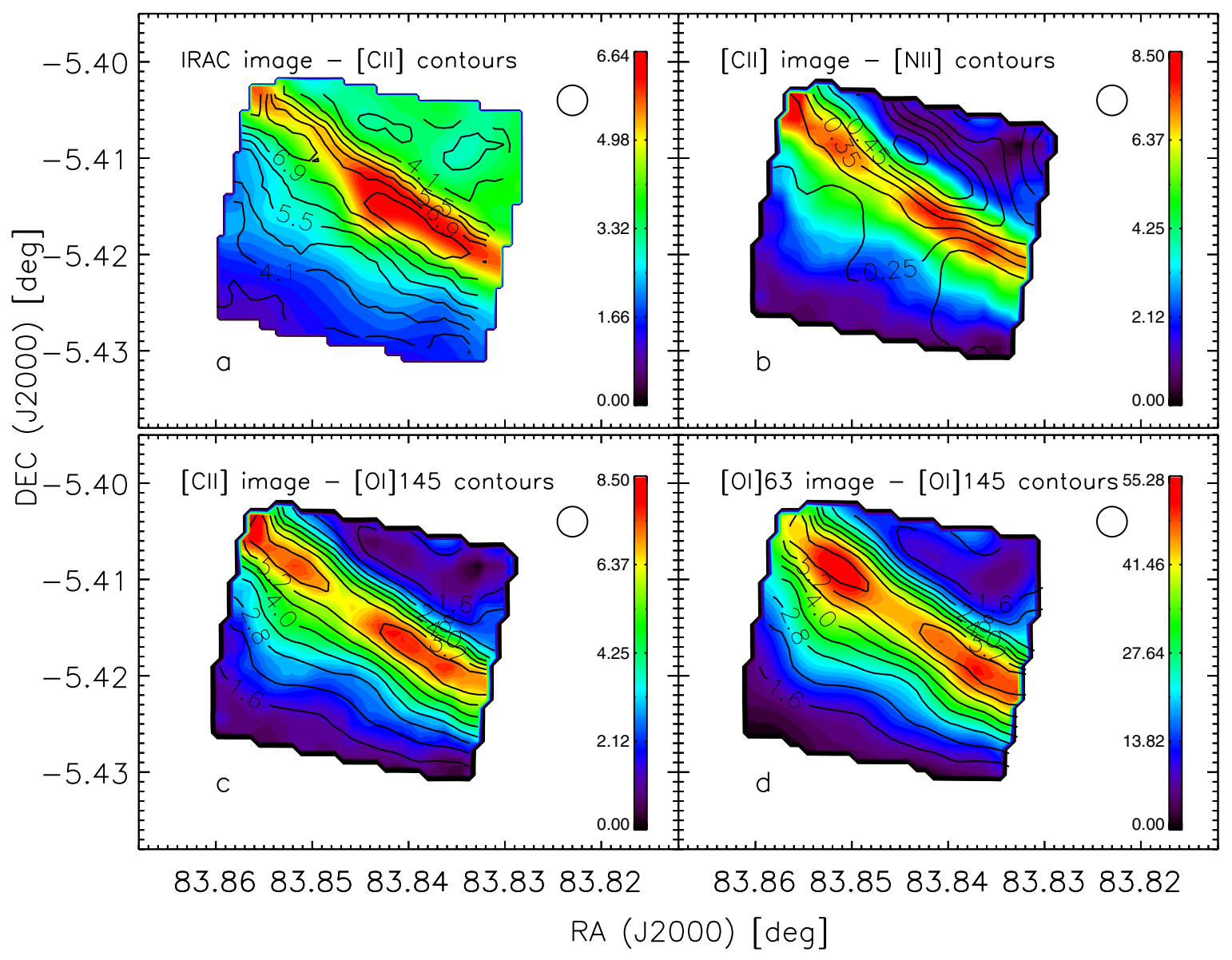

Fig. 3. Image and contour plots for different combination of lines and $8 \mu \mathrm{m}$ band as indicated in the title of each panel. All maps have been convolved to the PSF at $158 \mu \mathrm{m}$ and are in flux units of $10^{-6} \mathrm{~W} \mathrm{~m}^{-2} \mathrm{sr}^{-1}$ (except for the IRAC image which is in $10^{3} \mathrm{MJy} \mathrm{sr}^{-1}$ ). Here the intensity scales start at zero.

resolved and could result from the superposition of even smaller clumps. These knots could be clumps being photo-evaporated by the intense FUV penetrating the PDR. Gorti \& Hollenbach (2002) studied the effects of the radiation field on the evolution of clumps in PDRs. Using their relation between the size of the clumps and the column density of the FUV heated region $\left(N_{0}=\right.$ $2 \times 10^{21} \mathrm{~cm}^{-2}$, their Eq. (34)), we derive a density of the gas at the base of the photo-evaporating flow of $6-10 \times 10^{4} \mathrm{~cm}^{-3}$. This value is consistent with the inter-clump density we use in Sect. 5 for the modelling. Knowing that the density inside the clumps is higher than this, and assuming that the observed size is that of an isolated clump, we estimate that the photo-evaporation timescale of these clumps should be higher than $\sim 4500-7500 \mathrm{yr}$, depending on size.

This morphology is somewhat mimicked in the [O I] $145 \mu \mathrm{m}$ and [C II] $158 \mu \mathrm{m}$ maps, where the lower resolution has washed out the clumps of emission. As we move away from the Bar (both in front and behind) the emission decreases gradually. There is, however, a confined region of brighter emission in the north of the map (middle top of the [O I] $63 \mu \mathrm{m}$ map), probably due to an increase in density in the PDR which lies behind the $\mathrm{H}$ II region. Alternatively this could be the result of a geometrical effect where the inclination of the background PDR is steeper. This region is also revealed in the other three maps, albeit with less contrast. The [OI] $63 \mu \mathrm{m}$ map also points to an increased emission at the western edge of the Bar and extending north. This excess was also detected in $\mathrm{CO}$ emission from the ground (e.g., Lis et al. 1998).
The morphology of the [C $\mathrm{CI}]$ line follows very well that of the [O I] lines (especially that of [O I $145 \mu \mathrm{m}$ ) with the Bar peaking at the same position in the three maps. This is better seen in Figs. $3 \mathrm{c}$ and $\mathrm{d}$ where the images have been convolved to the same resolution as the $\left[\mathrm{C}_{\mathrm{II}}\right] 158 \mu \mathrm{m}$ map (largest PSF), and are shown with over-plotted contours. In Fig. $3 \mathrm{a}$ we also see that the PAH emission, as traced by the $8 \mu \mathrm{m}$ IRAC band, is close to the [C II] peak but slightly shifted towards the ionising star $\theta^{1}$ Ori C. The peak of $\left[\mathrm{C}_{\mathrm{II}}\right]$ emission in the top-left corner of the Bar is, however, not followed as well by the PAH emission. In Fig. 3d we can see that the different intensity levels in the $145 \mu$ m map delineate the Bar very well, whereas in the $63 \mu \mathrm{m}$ map the spread in the levels is broader. This is probably the result of the higher optical depth on the $63 \mu \mathrm{m}$ line, where changes in column density have a less pronounced effect than for the $145 \mu \mathrm{m}$ line.

The peak of the [N $\mathrm{NI}$ emission is displaced with respect to the other lines by about $12^{\prime \prime}$ towards to $\theta^{1}$ Ori C (Figs. 2 and $3 b$ ). This is expected because with an ionisation potential of $14.5 \mathrm{eV}$ the $[\mathrm{N}$ II] line comes from the ionised phase and is representative of the ionisation front. There is a small region of overlap between the $\left[\mathrm{N}_{\mathrm{II}}\right]$ and $\left[\mathrm{C}_{\mathrm{II}}\right]$ lines but this could be due to an orientation effect (Bar slightly tilted). We even note that the northeast tip (top-left) of the Bar that is seen in the [C II $158 \mu \mathrm{m}$ and [OI] $145 \mu \mathrm{m}$ maps (and also $8 \mu \mathrm{m}$ IRAC map, Figs. 1 and 2), delineates the boundary of emission of the [N II] line in that region. On the western side of the Bar, the $[\mathrm{N}$ II] emission is somewhat delineated by the above-mentioned emission extending north in the $\left[\mathrm{O}_{\mathrm{I}}\right] 63 \mu \mathrm{m}$ map and also detected in $\mathrm{CO}$ and 

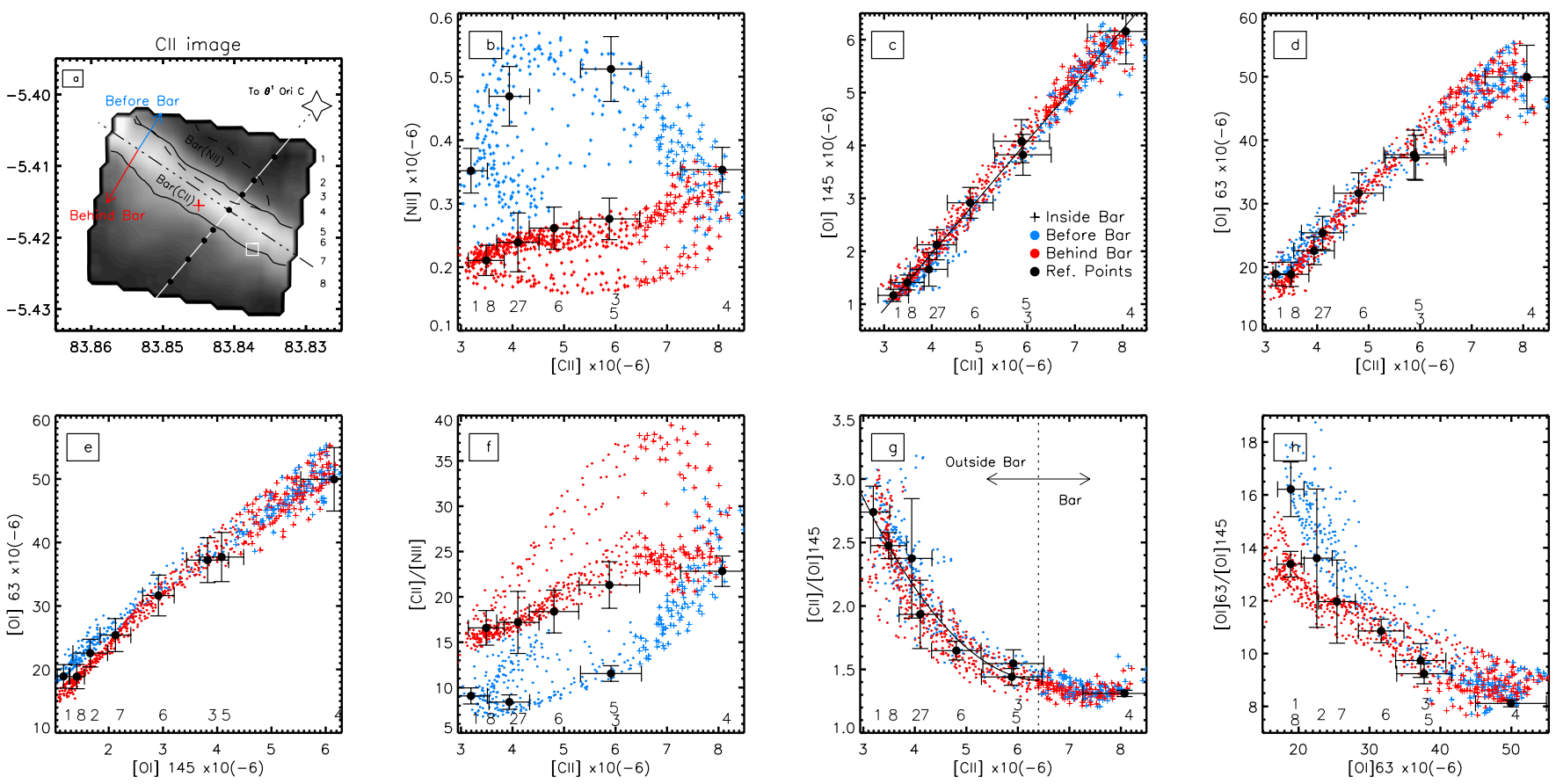

Fig. 4. Intensity plots for different combination of lines fluxes (in $10^{-6} \mathrm{~W} \mathrm{~m}^{-2} \mathrm{sr}^{-1}$ ) and ratios from the convolved maps. The different regions are indicated in the first panel and are labelled in the third, where blue represents points in front of the Bar and red behind the Bar. Plus symbols indicate points within the $\operatorname{Bar}\left(\mathrm{C}_{\text {II }}\right)$. The square data point is the position where the cooling is calculated (see Sect. 7). The reference points for the adopted cut (Sect. 5) are plotted in circles and include error bars to give an indication of the uncertainties at different regions. In panels c) and g) the solid lines represent respectively a linear fit $(y=-2.28+1.06 x)$, and a polynomial fit for the fainter region outside the Bar $\left(y=6.37-1.53 x+0.12 x^{2}\right)$.

dust emission. Finally, the gap in the center of the Bar, seen in the $\left[\mathrm{O}_{\mathrm{I}}\right]$ and $\left[\mathrm{C}_{\mathrm{II}}\right]$ lines, coincides with the maximum emission of the [N II] $122 \mu \mathrm{m}$ line in front of it (Fig. 3b). This could indicate that the radiation field in this specific spot is higher (or more intense) and has been able to photo-ionise the gas. Alternatively this could be due to variations in the column density.

\section{Line correlations and ratios}

For the purpose of the discussion we have divided the map into several regions which are represented in Fig. 4a and annotated in panel c of the same figure. Summarizing: data points in front of and behind the Bar are colour-coded in blue and red, respectively; plus symbols indicate points that trace the Bar in [C II] (those inside the solid contour line), and dots points outside the Bar in [C II]. In addition, selected points along an adopted cut (see Sect. 5) have been labeled (black dots) and include error bars.

Figure $4 \mathrm{~b}$ plots the $\left[\mathrm{N}\right.$ II] versus the $\left[\mathrm{C}_{\text {II }}\right]$ line fluxes. The points before and behind the Bar (blue and red respectively) occupy different regions but there are no clear trends. Abel et al. (2005) hinted, from their models, at a correlation between these two lines in the ionised medium. The intricate behaviour in our figure does not suggest that. On the other hand, Fig. 4c illustrates that the [C $\mathrm{II}] 158 \mu \mathrm{m}$ line follows very well the [O I $145 \mu \mathrm{m}$ line. A linear fit gives a slope of $1.059 \pm 0.005$. Variation of the [O I] $145 \mu \mathrm{m}$ line is dominated by the column density of the gas, and so this correlation indicates that most of the $[\mathrm{C} \mathrm{II}]$ comes from the PDR and not the $\mathrm{H}$ II region (see Sect. 6).

The [OI] $63 \mu \mathrm{m}$ and [C II] $158 \mu \mathrm{m}$ lines show also a good correlation (Fig. 4d) but it seems that the correlation outside the $\operatorname{Bar}\left(\mathrm{C}_{\text {II }}\right)$ differs with respect to the other regions. This is also seen in Fig. 4e where the two oxygen lines are plotted against each other. The points behind the Bar (red dots) are displaced compared to those before the Bar (blue dots), and there is a change of slope between points in the Bar (plus signs) and outside the Bar (dots). The excitation conditions of these lines are similar and therefore this behaviour could reflect the effect of opacities that more strongly affect the [OI]63 $\mu \mathrm{m}$ line (as it becomes self-absorbed at rather low column densities).

In the following panels we plot the ratio of several lines which, in an optically thin environment, allows us to remove the effect of column density in the trends:

- Figure 4f shows that the $\left[\mathrm{C}_{\mathrm{II}}\right](158 \mu \mathrm{m}) /\left[\mathrm{N}_{\mathrm{II}}\right](122 \mu \mathrm{m})$ ratio is different before and behind the Bar. A map of this line ratio is illustrated Fig. 5b where we clearly distinguish two regions, with the points before the Bar, which are more representative of the ionised gas, having a lower ratio. We find that a threshold of 15 for this ratio could be used to distinguish between emission from the neutral and ionised region.

- The $\left[\mathrm{C}_{\mathrm{II}}\right] 158 \mu \mathrm{m} /\left[\mathrm{O}_{\mathrm{I}}\right] 145 \mu \mathrm{m}$ ratio in Fig. $4 \mathrm{~g}$ decreases as we approach the Bar. The trend in the figure of the points outside the Bar (dots) can be approximated by a polynomial fit. This behaviour is again evident in the map ratio shown in Fig. 5a where points before and behind the Bar show the similar values. This suggests similar physical conditions in these two regions because the excitation parameters for both lines are different. This is not expected for a single PDR and suggests contamination of a background PDR (see Sect. 5) Another important point is that within the Bar the ratio is very homogeneous (within 16\%) but it differs from the regions outside the Bar. We can use the $\left[\mathrm{C}_{\mathrm{II}}\right] 158 \mu \mathrm{m} /\left[\mathrm{O}_{\mathrm{I}}\right] 145 \mu \mathrm{m}$ ratio to put a constraint on the densities by comparing with the predicted values from PDR models (Ferland et al. 1998; Kaufman et al. 1999). We find densities that vary from $10^{4}-10^{5} \mathrm{~cm}^{-3}$ in the Bar, and $10^{3}-10^{4} \mathrm{~cm}^{-3}$ outside it. 


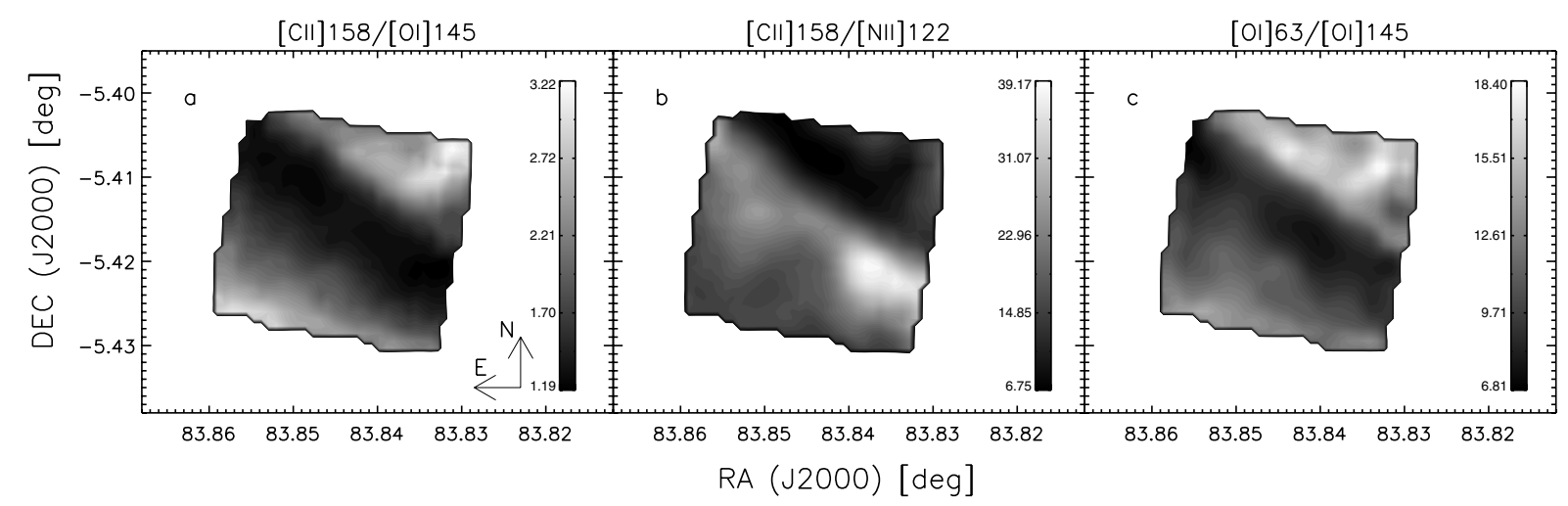

Fig. 5. Map ratios for different combination of lines as indicated in the title of each panel.

- In the last panel Fig. $4 \mathrm{~h}$, it is clear that the line ratio [O I] $63 \mu \mathrm{m} /\left[\mathrm{O}_{\mathrm{I}}\right] 145 \mu \mathrm{m}$ splits before and behind the Bar (being lower before the Bar). Given that the excitation conditions in these regions must be similar (Fig. $4 \mathrm{~g}$ ), we conclude that the observed difference is the result of [O I] $63 \mu \mathrm{m}$ becoming self-absorbed; in the Bar, as it is expected, the ratio is smaller because the column density is higher than outside the Bar. We note that the gas in the region where the [O I]63 $\mu \mathrm{m}$ line is optically thick must still be warm to excite the $\left[\mathrm{O}_{\mathrm{I}}\right] 145 \mu \mathrm{m}$ line $\left(E_{u}=326 \mathrm{~K}\right)$. From Fig. $5 \mathrm{c}$ we see that the ratio is indeed not homogeneous within the Bar (as it was for the [ $\left.\mathrm{C}_{\mathrm{II}}\right]$ and [O $\left.\mathrm{I}\right] 145 \mu \mathrm{m}$, Fig. 5a), indicating that opacity effects are present.

\section{Modelling}

We have modelled the line emission across the Bar using the radiative transfer code Cloudy (Ferland et al. 1998). This code computes the chemistry and radiative transfer at the surface of a molecular cloud (in our case assumed to be a planeparallel semi-infinite slab) illuminated by FUV photons from the ionising star(s). We adopt a Kurucz model for the star at $39600 \mathrm{~K}$ (Pellegrini et al. 2009), and ISM abundances (Savage \& Sembach 1996; Meyer et al. 1997, 1998). In order to reproduce the spatial stratification we adjust the starting point of the $\mathrm{H}$ II region and the density. Namely, the ionised region is adjusted to start at $0.134 \mathrm{pc}\left(67^{\prime \prime}\right)$ from the star. The adopted gas density is set to a constant value of $3200 \mathrm{~cm}^{-3}$ (Pellegrini et al. 2009) in the ionised region, and is then coupled with a profile density for the PDR as described in Arab et al. (in prep.), with a density scaled to $6 \times 10^{4} \mathrm{~cm}^{-3}$ at the peak of the [O I $]$ and $\left[\mathrm{C}_{\mathrm{II}}\right]$ and emission (projected distance of $0.246 \mathrm{pc}$ ). No tilting is assumed for the Bar and the depth of the PDR along the line of sight is adjusted to $0.35 \mathrm{pc}$.

To illustrate the comparison between the observed fluxes and the model (convolved to the $158 \mu \mathrm{m}$ PSF) we assume the cut illustrated in Fig. 4a. This cut is also adopted for the discussion in our accompanying papers by Habart et al. (in prep.) and Arab et al. (in prep.). It is made to pass through the exciting star $\theta^{1}$ Ori $C\left(5^{\mathrm{h}} 35^{\mathrm{m}} 16.46^{\mathrm{s}},-5^{\mathrm{d}} 23^{\mathrm{m}} 23.17^{\mathrm{s}}\right)$ and an arbitrary point in the $\operatorname{Bar}\left(5^{\mathrm{h}} 35^{\mathrm{m}} 21.82^{\mathrm{s}},-5^{\mathrm{d}} 24^{\mathrm{m}} 59.18^{\mathrm{s}}\right)$. The chosen point in the Bar minimizes effects of other contaminating stars in the direction of the cut that can affect the dust (Arab et al., in prep.), and allows us to sample different conditions in the region. In Fig. 6 the solid line represents the output of the model, and the
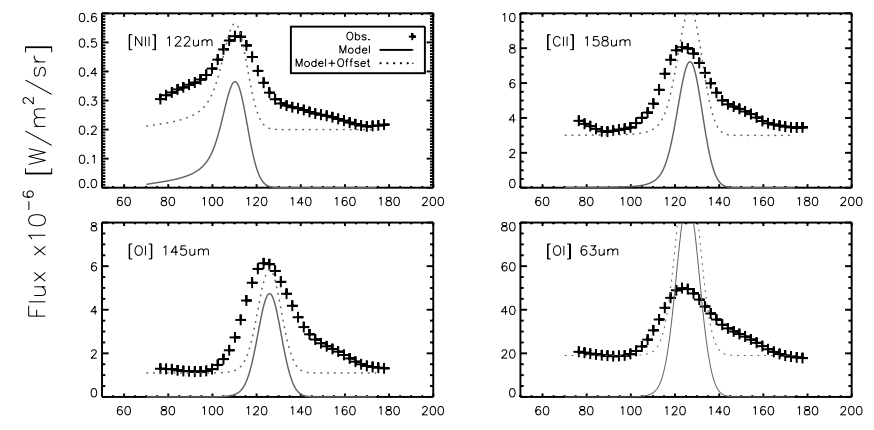

Rel. Position - Star (arcsec)

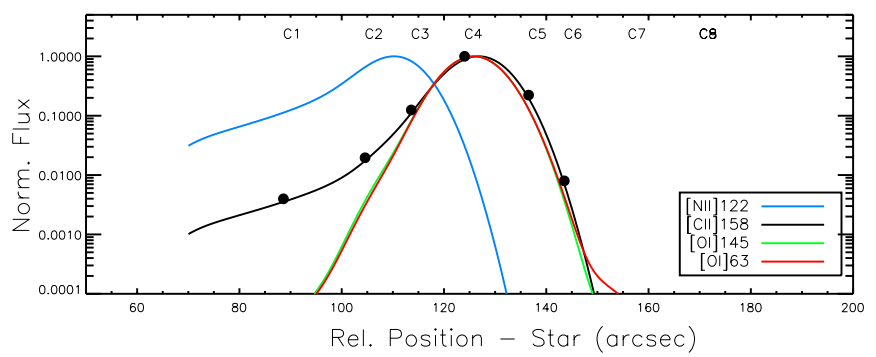

Fig. 6. Observed (plus symbols) and modelled (solid and dotted lines) profiles along the cut shown in Fig. 4 (see Sect. 5) measured with respect to the distance to the exciting star $\theta^{1}$ Ori C. Observed and modelled fluxes are convolved to the $158 \mu \mathrm{m}$ PSF, where the dotted line includes an offset due to background emission (see text). The first four panels compare for each line the observed convolved profiles with a Cloudy model, where this has been scaled to the peak of the [O I] $(145 \mu \mathrm{m})$ emission. The bottom panel compares the normalized model profiles. In this panel, the solid dots represent the position of the reference points in the adopted cut (Fig. 4a).

dotted line the model plus an offset (see point 3 ). From the comparison in Fig. 6 we find that:

1. This simple model does a very good job in reproducing the observed spatial stratification of the peak positions of the

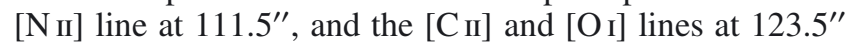
(top and middle panels). This stratification is the result of the attenuation of the incident radiation field across the Bar and is sensitive to the density profile.

2. The model also shows a gradual increase of the [N II] line as we approach the Bar (better seen in the bottom panel) which is also seen in the observations, while in contrast the oxygen lines show a more abrupt increase. This is the result 
of the ionisation structure. The model also shows a gradual increase for the [C $\mathrm{II}]$ line but this is not clear from the observations. This could be the result of it being more easily excited (low temperature and density) compared to the oxygen lines and/or contribution of the $\left[\mathrm{C}_{\mathrm{II}}\right]$ line from the ionised phase.

3. It is striking that the observed profiles do not fall to zero as the model (solid lines) does both before and after the Bar. This background emission is significant, amounting to about $\sim 18 \%$ of the peak emission for the [O I] $145 \mu \mathrm{m}$ line and $\sim 38 \%$ for the other three lines. These offsets have been added to the model emission as the dotted lines in the figure. This suggests the presence of another PDR(s), probably from the background cavity in the molecular cloud, which must produce the base of emission that we measure. This PDR is probably not entirely face-on because the strong intensity observed, which may result from a limb brightening effect, cannot be reproduced by a face-on PDR model.

4. The model line profiles are narrower than the observations. This could be due to the Bar being tilted to the observer $\left(\$ 10^{\circ}\right)$, an effect already inferred in other studies (e.g., Pellegrini et al. 2009). Alternatively, a highly structured medium would allow FUV radiation to permeate the region and heat gas on larger spatial scales.

5. In order to reproduce the absolute flux, and assuming that the base emission from the additional PDR(s) has an additive effect on top of the emission of the Bar (dotted line), we need to assume a depth of the PDR in the model of $0.35 \mathrm{pc}$. The depth given above is larger than, but comparable to, the width of the Bar $(\sim 0.06 \mathrm{pc})$ as expected for a tilted edge-on Bar.

6. In this model we take into account optical depth effects across the $\mathrm{PDR}^{4}$ but not along the line of sight. However, we can see that the model (with offset) reproduces the peak of emission of the [N II] $122 \mu \mathrm{m}$, [O I $] 145 \mu \mathrm{m}$ fairly well, and the $\left[\mathrm{C}_{\mathrm{II}}\right] 158 \mu \mathrm{m}$ within factor 1.2. This indicates that, except for the [O I] $63 \mu \mathrm{m}$ line, the emission of these lines are not dominated by optical effects.

A more detailed modelling of the region including additional $\mathrm{PDR} / \mathrm{s}$ (even the effects of clumpiness and tilting) and dust emission is outside our scope here but will be presented in a future paper. Still, it is encouraging to see how a simple model (single edge-on PDR) can be used to at least reproduce with a fair degree of success the observations of such a complex environment.

\section{6. [C II] from the PDR and $\mathrm{H} I I$ region}

In the ISM, the $\left[\mathrm{C}_{\mathrm{II}}\right] 158 \mu \mathrm{m}$ line is important in the study of the cooling and chemistry of PDRs. In extragalactic studies it is important for redshift determinations, and for the extent to which its luminosity is a measure of the star formation rate (SFR, Stacey et al. 1991; Meijerink et al. 2007; Luhman et al. 2003). With a low ionisation potential of $11.3 \mathrm{eV}$, this line can originate both in the PDR and in the HiI region. It is thus important to characterise in detail its contribution in different environments. Because of the high radiation field and high density in the Orion nebula, one expects that most of the $\left[\mathrm{C}_{\mathrm{II}}\right]$ should originate from the PDR. The PACS maps show in great detail the distribution of the $[\mathrm{C}$ II] and $[\mathrm{N}$ II] lines where we can investigate this issue.

We have therefore quantified the contribution of [C $\mathrm{CII}_{\mathrm{I}} 158 \mu \mathrm{m}$ and [N $\mathrm{NI}_{\text {II }} 122 \mu \mathrm{m}$ to the Bar as traced by the

\footnotetext{
4 We assume both thermal and micro-turbulence line width with a turbulence velocity of $3 \mathrm{~km} \mathrm{~s}^{-1}$.
}

[C II] emission $\left(\operatorname{Bar}\left(\mathrm{C}_{\mathrm{II}}\right)\right)$, and as traced by the $\left[\mathrm{N}_{\mathrm{II}}\right]$ emission $\left(\mathrm{Bar}\left(\mathrm{N}_{\text {II }}\right)\right)$. These are represented as areas enclosed by the solid and dashed lines, respectively, in Fig. 4a. This combined region, which we name $\operatorname{Bar}\left(\mathrm{C}_{\mathrm{II}}+\mathrm{N}_{\text {II }}\right)$, encompasses the peak emission of the PDR and ionised region. We find that $76 \%$ of the $[\mathrm{C} I \mathrm{II}]$ emission is coming from the PDR region $\left(\operatorname{Bar}\left(\mathrm{C}_{\mathrm{II}}\right)\right.$ ) relative to the total emission in the $\mathrm{Bar}\left(\mathrm{C}_{\mathrm{II}}+\mathrm{N}\right.$ II $)$. The remaining $31 \%$ comes from the $\operatorname{Bar}\left(\mathrm{N}_{\text {III }}\right)$ region ${ }^{5}$. This $31 \%$ contribution to the $\operatorname{Bar}\left(\mathrm{N}_{\text {II }}\right)$ region could include emission from the ionised region or from the additional PDR (see Sect. 5). To estimate this, and ignoring confusion from tilting effects, we can use the close relation of the $\left[\mathrm{C}_{\mathrm{II}}\right]$ line with the $\left[\mathrm{O}_{\mathrm{I}}\right] 145 \mu \mathrm{m}$ line. For a broad range of densities between $10^{3}$ and $10^{5}$ (typical of PDRs), the $\left[\mathrm{C}_{\mathrm{II}}\right] /\left[\mathrm{O}_{\mathrm{I}}\right] 145 \mu \mathrm{m}$ ratio varies between 0.5 and 2 (Ferland et al. 1998; Kaufman et al. 1999; Le Petit et al. 2006). The observed [OI] $145 \mu \mathrm{m}$ in the $\operatorname{Bar}\left(\mathrm{N}_{\mathrm{II}}\right)$ is about $2 \times 10^{-6} \mathrm{~W} \mathrm{~m}^{-2} \mathrm{sr}^{-1}$, from which we predict a PDR $\left[\mathrm{C}_{\mathrm{II}}\right]$ emission between 1 to $4 \times 10^{-6} \mathrm{~W} \mathrm{~m}^{-2} \mathrm{sr}^{-1}$. We can then compare this range to our observed [ $\mathrm{C}_{\mathrm{II}}$ ] emission of $4 \times 10^{-6} \mathrm{~W} \mathrm{~m}^{-2} \mathrm{sr}^{-1}$ in this region. Thus, from the original $31 \%$ of $\left[\mathrm{C}_{\mathrm{II}}\right]$ in this region, we deduce that either all could come from the additional PDR, or the $\mathrm{H}$ II region could contribute to at most $24 \%$.

Following the analysis by Oberst et al. (2011) in the Carina nebula, we have compared the expected $\left[\mathrm{C}_{\mathrm{II}}\right] /\left[\mathrm{N}_{\mathrm{II}}\right]$ ratio in the ionised medium with the observed values in the $\mathrm{Bar}\left(\mathrm{N}_{\text {II }}\right)$ region. This can be used to get an additional estimate of the contribution from the ionised region to the $\left[\mathrm{C}_{\mathrm{II}}\right]$ line. To do this we have complemented our observations with the SPIRE-FTS data of the $\left[\mathrm{N}_{\mathrm{II}}\right] 205 \mu \mathrm{m}$ line (Habart et al., in prep.), and have calculated the theoretical curves for the $\left[\mathrm{N}_{\text {II }}\right] 122 \mu \mathrm{m} /\left[\mathrm{N}_{\text {III }}\right] 205 \mu \mathrm{m}$, $\left[\mathrm{C}_{\mathrm{II}}\right] 158 \mu \mathrm{m} /\left[\mathrm{N}_{\mathrm{II}}\right] 205 \mu \mathrm{m}$, and [C $\left.\mathrm{II}\right] 158 \mu \mathrm{m} /[\mathrm{N} \mathrm{II}] 122 \mu \mathrm{m}$ ratios at a temperature of $9000 \mathrm{~K}$ (Nieva \& Simón-Díaz 2011). The $[\mathrm{C}$ II $] /[\mathrm{N}$ II $]$ line ratios depend on the relative ionic abundance of $\mathrm{C}_{\text {II }}$ and $\mathrm{N}_{\text {III. }}$ We use the carbon and nitrogen abundances adopted in Sect. 5, coupled with the ionisation fraction of

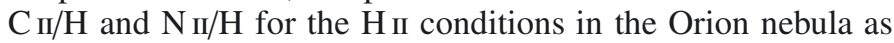
given by Rubin (1985), to derive a relative ionic abundance of $[\mathrm{C}$ II/N II $] \sim 1.6$. These theoretical curves are shown in Fig. $7^{6}$. The $\left[\mathrm{N}_{\text {II }}\right] 122 \mu \mathrm{m} /\left[\mathrm{N}_{\text {II }}\right] 205 \mu \mathrm{m}$ ratio can be used to determine the electron density. The observed ratio (square in the figure) falls in the non-linear regime of the theoretical curve (solid line) but can serve to obtain an upper limit in the density of $1000 \mathrm{~cm}^{-3}$, consistent with our adopted value of $3200 \mathrm{~cm}^{-3}$ (Pellegrini et al. 2009). The observed [C $\mathrm{C}_{\text {II }} 158 \mu \mathrm{m} /\left[\mathrm{N}_{\text {II }}\right] 205 \mu \mathrm{m}$, and $\left[\mathrm{C}_{\mathrm{II}}\right] 158 \mu \mathrm{m} /\left[\mathrm{N}_{\mathrm{II}}\right] 122 \mu \mathrm{m}$ line ratios, grey areas in the figure, are clearly above the theoretical curves (dotted and dashed lines respectively). Taking into account the uncertainties in the line fluxes (enclosing boxes in the figure), the observed ratios indicate that no more than $9 \%$ of the $[\mathrm{C}$ II $] 158 \mu \mathrm{m}$ comes from the ionised region. Allowing for an uncertainty of about a factor 2 in the relative abundance of $\mathrm{C}_{\text {II }} / \mathrm{N}_{\text {II }}$ and collisional strengths used to calculate the theoretical curves, we can safely conclude that in the Bar less than $18 \%$ of the [ $\mathrm{C}_{\mathrm{II}}$ ] emission originates in the ionised medium. This is a slightly lower threshold than our previous estimate.

Abel et al. (2005) made some modifications to the Cloudy code to, among others things, estimate the contribution of several PDR lines from the $\mathrm{H}_{\text {II }}$ region. They apply their model to the

\footnotetext{
5 These quantities do not add to $100 \%$ because there is an overlapping region as it is explained in Sect. 3.

${ }^{6}$ The $\left[\mathrm{C}_{\mathrm{II}}\right] 158 \mu \mathrm{m} /[\mathrm{N}$ II] $(122,205 \mu \mathrm{m})$ curves can be applied to any other object by simply scaling them to the ratio between the relative abundance of $\left[\mathrm{C}_{\mathrm{II}} / \mathrm{N}_{\mathrm{II}}\right]$ in the object and that assumed here (1.6).
} 


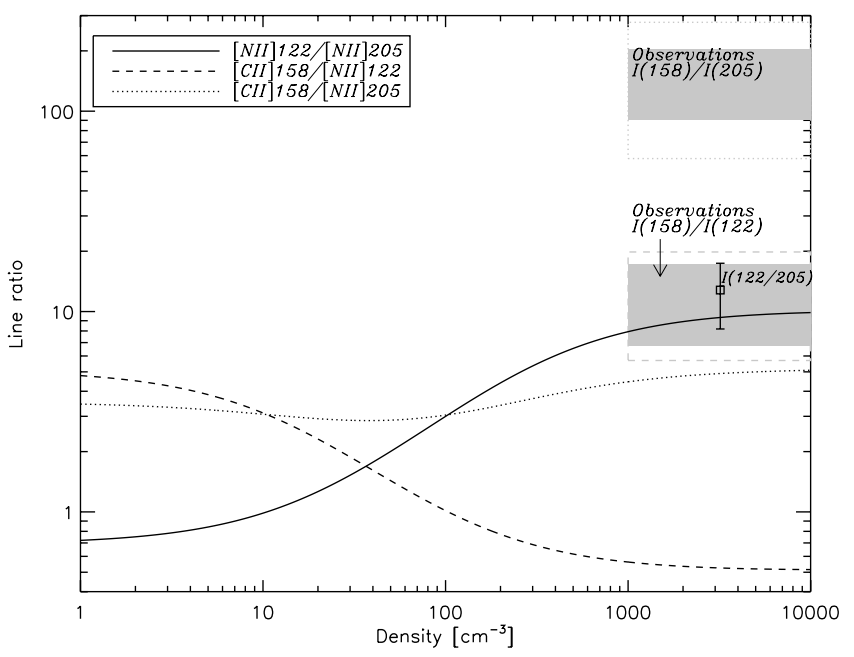

Fig. 7. Line intensity ratios for different combinations of the [N $\mathrm{NI}]$ (122 and $205 \mu \mathrm{m}$ ) and [C $\mathrm{II}] 158 \mu \mathrm{m}$ lines. Theoretical curves are shown by the solid, dotted, and dashed lines. Grey areas indicate the range of observed ratios, and the enclosing boxes represent the range of uncertainties in the ratios. The square point is the ratio of the [N II] 122/205 lines.

starburst galaxy NGC 253 where they find that about $30 \%$ of the [C II] comes from the ionised medium. More recently, Mookerjea et al. (2011) using PACS data have mapped the FIR emission of the [C II] $158 \mu \mathrm{m}$ line in spiral galaxy M33. They find that between 20 to $30 \%$ of this emission comes from the ionised medium. Their values are slightly higher than our determinations, which is not surprising since we are probing dense PDR in the Orion Bar while in these galaxies lower density $\mathrm{H}_{\text {II }}$ regions could dominate the emission. We note, however, that this comparison is hampered by the difference between the larger scales (where different regions are mixed) that these studies probe, and the small scales probed in our study.

\section{Cooling}

In order to establish the role of the $\left[\mathrm{CII}_{\mathrm{II}}\right]$ and $\left[\mathrm{O}_{\mathrm{I}}\right]$ lines in the cooling of the PDR we have compared their contribution to that of other relevant cooling lines in the mid- and FIR. We have included in this calculation the $\mathrm{H}_{2}$ rotational lines from the ISO/SWS, and ${ }^{12} \mathrm{CO}$ rotational lines $(J=4-3$ to $J=21-20)$, ${ }^{13} \mathrm{CO}(J=5-4$ to $J=14-13), \mathrm{H}_{2} \mathrm{O}$, and $\mathrm{CH}^{+}$from the PACS and SPIRE instruments (Habart et al. 2010; Joblin et al., in prep.). To make this comparison we use the same position $\left(5^{\mathrm{h}} 35^{\mathrm{m}} 21^{\mathrm{s}},-5^{\circ} 25^{\prime} 18^{\prime \prime}\right)$ of the $\mathrm{H}_{2}$ measurements by ISO to derive the contribution of each line (or cascade of lines). This position is indicated by the white square in Fig. 4a. For this comparison we convolve all the data to the largest beam size (SPIRE 40"). We find that in this region the $\left[\mathrm{C}_{\mathrm{II}}\right]$ and $\left[\mathrm{O}_{\mathrm{I}}\right]$ lines studied in this paper contribute $90 \%$ of the total power emitted by all these lines, with the [OI] $63 \mu \mathrm{m}$ line contributing $72 \%$ of this emission. The $\mathrm{CO}, \mathrm{H}_{2}$, and $\mathrm{CH}^{+}$contribute, respectively, 5, 4, and less than $1 \%$. These estimates accentuates the importance of the [O I] $63 \mu \mathrm{m}$ line to the cooling budget from the gas lines in these regions. This fact is also highlighted by the relative strength of this line compared to the other lines in the region (see Fig. 6).

\section{Summary and conclusions}

We have presented the first HSO observations of the [C II] $158 \mu \mathrm{m}$, [O I $63 \mu \mathrm{m}$ and $145 \mu \mathrm{m}$, and [N $\left.{ }_{\mathrm{II}}\right] 122 \mu \mathrm{m}$ lines of the Orion Bar. Its angular resolution has allowed us to map the spatial distribution of these lines in unprecedented detail.

The $\left[\mathrm{C}_{\mathrm{II}}\right]$ and $\left[\mathrm{O}_{\mathrm{I}}\right]$ maps peak at the same position and fall close to the peak emission of PAHs (as traced by the Spitzer/IRAC $8 \mu \mathrm{m}$ band). The [N II] peaks slightly closer to $\theta^{1}$ Ori $\mathrm{C}$ with a small region of overlap with respect to the other PDR lines. Within the Bar we can distinguish between knots (clumps) of emission, about $0.01-0.02 \mathrm{pc}$ in size (6 to $\left.10^{\prime \prime}\right)$, and which are $16 \%$ higher in flux than the interclump medium. These clumps could be photo-evaporated by the FUV inside the PDR. These knots of emission are better seen in the [O I] $63 \mu \mathrm{m}$ map as this line offers the best resolution (having the smallest PSF), it is also seen in the $\left[\mathrm{O}_{\mathrm{I}}\right] 145 \mu \mathrm{m}$ and $\left[\mathrm{C}_{\mathrm{II}}\right] 158 \mu \mathrm{m}$ maps.

The $\left[\mathrm{C}_{\mathrm{II}}\right] 158 \mu \mathrm{m}$ correlates very well with the [O I] $145 \mu \mathrm{m}$ emission. The [C II $] 158 \mu \mathrm{m}$ line does not correlate with the [N II] in the ionised region as some studies have suggested. The combined information on the $[\mathrm{NII}]$ and $\left[\mathrm{O}_{\mathrm{I}}\right]$ lines provides a great diagnostic to estimate the emission from the [C II $] 158 \mu \mathrm{m}$ line, and to distinguish between an origin in an ionised or neutral region. The ratio between the [O I] $145 \mu \mathrm{m} / 63 \mu \mathrm{m}$ lines show the effect of the opacities, where the [O I] $63 \mu \mathrm{m}$ line becomes selfabsorbed at high column densities.

We have modelled the emission of the lines with the photoionisation code Cloudy and reproduce the relative position of the lines. The emission profiles reveal that, in addition to the Bar, there is a significant background emission all over the region (present for all four lines). This points to the presence of additional PDR(s). This should be follow up with detail radiative transfer models to infer the physical conditions and coupled to what has been learned from the emission of dust.

The $[\mathrm{CII}]$ line can come from the neutral and ionised medium. We have made different estimations of its contribution and find that most of the $\left[\mathrm{C}_{\mathrm{II}}\right]$ emission originates in the PDR(s) $(>82 \%)$. Using ancillary ISO and Herschel data we have calculated the total power emitted by the most relevant cooling lines from the mid- to FIR ([O I], [C II], $\mathrm{CO}, \mathrm{H}_{2} \mathrm{O}, \mathrm{CH}^{+}$). We show that the power emitted by the atomic [C $\mathrm{CII}_{\mathrm{II}} 158 \mu \mathrm{m}$, and [O I] $63 \mu \mathrm{m}$ and $145 \mu \mathrm{m}$ lines account for $90 \%$ of the power emitted in the region by all of the cooling lines considered, with the [O I] $63 \mu \mathrm{m}$ line contributing $72 \%$ of the total. This emphasizes the predominant role of the latter in the cooling process from emission lines of these regions.

Acknowledgements. We would like to thank the referee, David Hollenbach for his comments and suggestions. J.B.S. wishes to acknowledge the support from a Marie Curie Intra-European Fellowship within the 7th European Community Framework Program under project number 272820. HCSS, HSpot, and HIPE are joint developments by the Herschel Science Ground Segment Consortium, consisting of ESA, the NASA Herschel Science Center, and the HIFI, PACS and SPIRE consortia. J.B.S. thanks E. Romano-Diaz for help with contour manipulation. We thank V. Lebouteiller for the use of the PACSman software and discussion on data reduction.

\section{References}

Abel, N. P., Ferland, G. J., Shaw, \& van hoof, P. A. M. 2005, ApJS, 161, 65 Abergel, A., Arab, H., Compiegne, M., et al. 2010, A\&A, 518, L96 Bernard-Salas, J., \& Tielens, A. G. G. M. 2005, A\&A, 431, 523 Burton, M. G., Hollenbach, D. J., \& Tielens, A. G. G. M. 1990, ApJ, 365, 620 Ferland, G. J., Korista, K. T., Verner, D. A., et al. 1998, PASP, 110, 761 
Gorti, U., \& Hollenbach, D. 2002, ApJ, 573, 215

Habart, E., Dartois, E., Abergel, A., et al. 2010, A\&A, 518, L116

Habing, H. J. 1968, Bull. Astr. Inst. Netherlands, 19, 421

Herrmann, F., Madden, S. C., Nikola, T., et al. 1997, ApJ, 481, 343

Hollenbach, D. J., \& Tielens, A. G. G. M. 1999, Rev. Mod. Phys., 71, 173

Kaufman, M. J., Wolfire, M. G., Hollenbach, D. J., \& Luhman, M. L. 1999, ApJ,

527,795

Lis, D. C., Serabyn, E., Keene, J., et al. 1998, ApJ, 509, 299

Le Petit, F., Nehmé, C., Le Bourlot, J., \& Roueff, E. 2006, ApJS, 164, 506

Lerate, M. R., Barlow, M. J., Swinyard, J. R., et al. 2006, MNRAS, 370, 597

Luhman, M. L., Satyapal, S., Fischer, J., et al. 2003, ApJ, 594, 758

Marconi, A., Tesli, L., Natta, A., \& Walmsley, C. L. 1998, A\&A, 330, 696

Meijerink, R., Spaans, M., \& Israel, F. P. 2007, A\&A, 461, 793

Menten, K. M., Reid, M. J., Forbrich, J., \& Brunthaler, A. 2007, A\&A, 474, 515

Meyer, D. M., Cardelli, J. A., \& Sofia, U. J. 1997, ApJ, 490, 103

Meyer, D. M., Jura, M., \& Cardelli, J. A. 1998, ApJ, 493, 222

Mookerjea, B., Kramer, C., Buchbender, C., et al. 2011, A\&A, 532, A152
Naylor, D. A., Dartois, E., Habart, E., et al. 2010, A\&A, 518, L117

Nieva, M.-F., \& Simón-Díaz, S. 2011, A\&A, 532, A2

Oberst, T. E., Parshely, S. C., Nikola, T., et al. 2011, ApJ, 739, 100

Ott, S. 2010, ASP Conf. Ser., 434, 139

Pellegrini, E. W., Bladwin, J. A., Ferland, G. J., Shaw, G., \& Heathcote, S. 2009, ApJ, 693, 285

Pilbratt, G. J., Riedinger, J. R., Passvogel, T., et al. 2010, A\&A, 518, L1

Poglitsch, A., Waelkens, C., Geis, N., et al. 2010, A\&A, 518, L2

Rubin, R. H. 1985, ApJS, 57, 349

Rubin, R. H., Simpson, J. P., O’Dell, C. R., et al. 2011, MNRAS, 410, 1320

Savage, B. D., \& Sembach, K. R. 1996, ARA\&A, 34, 279

Stacey, G. J., Geis, N., Genzel, R., et al. 1991, ApJ, 382, 37

Tielens, A. G. G. M., \& Hollenbach, D. 1985, ApJ, 291, 722

Tielens, A. G. G. M., Meixner, M. M., van der Werf, P. P., et al. 1993, Science, 262,86

van der Werf, P. P., Stutzki, J., Sternberg, A., \& Krabber, A. 1996, A\&A, 313, 633 